\title{
Density Variations over Subparsec Scales in Diffuse Molecular Gas
}

\author{
K. $\operatorname{Pan}^{1,2}$, S. R. Federman ${ }^{1,2}$, and D. E. Welty ${ }^{3,4}$
}

\begin{abstract}
We present high-resolution observations of interstellar $\mathrm{CN}, \mathrm{CH}, \mathrm{CH}^{+}, \mathrm{Ca} \mathrm{I}$, and Ca II absorption lines toward the multiple star systems HD206267 and HD217035. Substantial variations in CN absorption are observed among three sight lines of HD206267, which are separated by distances of order 10,000 $\mathrm{AU}$; smaller differences are seen for $\mathrm{CH}, \mathrm{CH}^{+}$, and $\mathrm{Ca}$ I. Gas densities for individual velocity components are inferred from a chemical model, independent of assumptions about cloud shape. While the component densities can differ by factors of 5.0 between adjacent sightlines, the densities are always less than 5000 $\mathrm{cm}^{-3}$. Calculations show that the derived density contrasts are not sensitive to the temperature or reaction rates used in the chemical model. A large difference in the $\mathrm{CH}^{+}$profiles (a factor of 2 in column density) is seen in the lower density gas toward HD217035.
\end{abstract}

Subject headings: ISM: clouds — ISM: structure — ISM: molecules — stars: individual (HD206267, HD217035)

\section{Introduction}

Evidence for pervasive subparsec-scale structure in the diffuse interstellar medium (ISM) has been accumulating over the past three decades through radio and optical/ultraviolet observations. Observations of H I $21 \mathrm{~cm}$ absorption toward high-velocity pulsars (Frail et al. 1994) and toward extended extragalactic radio sources (Faison \& Goss 2001) reveal variations in optical depth on scales of 10 to 100 AU. Radio observations of absorption from

\footnotetext{
${ }^{1}$ Department of Physics and Astronomy, University of Toledo, Toledo, OH 43606.

${ }^{2}$ Guest Observer, McDonald Observatory, University of Texas at Austin.

${ }^{3}$ Astronomy and Astrophysics Center, University of Chicago, 5640 South Ellis Ave., Chicago, IL 60637.

${ }^{4}$ Visiting Observer, Kitt Peak National Observatory, National Optical Astronomy Observatories, which is operated by AURA, Inc., under cooperative agreement with the National Science Foundation.
} 
$\mathrm{H}_{2} \mathrm{CO}$ and $\mathrm{OH}$ toward extragalactic sources (Moore \& Marscher 1995) also show changes in optical depth over time. Multi-epoch optical measurements provide another probe of these small-scale structures (e.g., Lauroesch, Meyer, \& Blades 2000; Price, Crawford, \& Barlow 2000; Crawford et al. 2000; Welty \& Fitzpatrick 2001). On intermediate scales of $10^{2}$ to $10^{5} \mathrm{AU}$, optical/ultraviolet observations of interstellar absorption lines (mainly from the trace species Na I and K I) toward individual members of binary or multiple star systems (e.g., Meyer \& Blades 1996; Watson \& Meyer 1996; Lauroesch et al. 1998; Lauroesch \& Meyer 1999-LM99) suggest ubiquitous structure. Structure on these scales is also implied by variations of Na I and K I absorption profiles toward members of clusters (e.g., Langer, Prosser, \& Sneden 1990; Bates et al. 1995) and toward bright extended cores of globular clusters (Meyer \& Lauroesch 1999; Andrews, Meyer, \& Lauroesch 2001). In this Letter, we

present optical spectra at a resolution of $\sim 1.7 \mathrm{~km} \mathrm{~s}^{-1}$ which reveal differences in molecular profiles across two multiple star systems.

While it is well established that the column densities of some atomic (H I, Na I, K I) and molecular $\left(\mathrm{H}_{2} \mathrm{CO}, \mathrm{OH}\right)$ species vary on scales of 10 to $10^{5} \mathrm{AU}$ in diffuse sight lines, detailed information on the physical conditions causing the variations is generally not available. A key question posed by the observations is whether the subparsec structure is caused by density variations (e.g., Frail et al. 1994; LM99; Crawford et al. 2000), by fluctuations in ionization equilibrium (LM99; Welty \& Fitzpatrick 2001), by the geometric structure of clouds (Heiles 1997), or by something else. A crucial point is to determine accurate physical conditions for the gas showing variations in column density. As a step in this direction, we present high resolution absorption spectra of $\mathrm{CN}, \mathrm{CH}, \mathrm{CH}^{+}, \mathrm{Ca} \mathrm{I}$ and Ca II, discuss the observed velocity component structure, and derive gas densities of components based on a simplified chemical model for $\mathrm{CH}, \mathrm{C}_{2}$, and $\mathrm{CN}$ (Federman et al. 1994; Knauth et al. 2001). We also investigate the sensitivity of the derived densities on cloud temperature and chemical reaction rates.

\section{Observations and Data Reduction}

High resolution spectra were obtained for two multiple star systems in the active star-forming regions Cep OB2 and OB3 (Table 1). Most of the observations were acquired in 2000 September with the 2d-coudé spectrograph on the $2.7 \mathrm{~m}$ telescope at McDonald Observatory (Tull et al. 1995). A cross-disperser and the availability of a thin Textronix $2048 \times 2048 \mathrm{CCD}$ made it possible to get $\mathrm{CN}, \mathrm{CH}, \mathrm{CH}^{+}, \mathrm{Ca}$ I, and $\mathrm{Ca}$ II features in a single exposure. In order to achieve a final signal-to-noise $(\mathrm{S} / \mathrm{N})$ ratio greater than 50 , five 30-min exposures were obtained for each target. Comparison spectra from a Th-Ar hollow cathode 
lamp were taken every two hours, while biases and flat fields were acquired each night. Dark frames were also obtained as a check on the amount of thermal noise from the cooled CCD. The setup yielded a spectral resolution of $\sim 1.7 \mathrm{~km} \mathrm{~s}^{-1}$ which was determined from the widths of the Th-Ar lines. The star HD206267A was observed in 1995 with the $0.9 \mathrm{~m}$ Coudé Feed Telescope at Kitt Peak National Observatory. Spectra for K I, Ca II, CN, and $\mathrm{CH}$ were obtained using camera 6 , the echelle grating, and the F3KB CCD, at resolutions of about $1.3 \mathrm{~km} \mathrm{~s}^{-1}$. Comparison of K I spectra of HD206267A obtained in 1995, 1998, and 2001 reveal no obvious temporal variation.

The raw images were reduced in the usual manner with the NOAO IRAF echelle data reduction package. The individual frames were first bias-subtracted. Cosmic-ray hits and scattered light were removed from each stellar image and flat field. The flat fields were divided into the stellar images to account for differences in pixel-to-pixel sensitivity. Since each echelle order was a few pixels wide, the orders were summed across this width. Then all extracted one-dimensional spectra were calibrated in wavelength using the Th-Ar comparison spectra. After correcting for Doppler motion, individual spectra for each sight line were combined. Finally, the combined spectra were normalized to unity using low-order polynomials. Final spectra appear in Figures 1 and 2; their S/N ratios range from 50 to 150.

Component structure is clearly seen in the spectra. The equivalent width, $W_{\lambda}$, for each velocity component was determined by Gaussian fit. We attempted to use the same velocity structure to fit all the absorption features in all lines of sight in each stellar system. While very good fits for HD217035A/B and HD206267A/C were obtained with essentially the same component structures, a slightly different component structure was required to fit the spectra of HD206267D. For each sightline, however, the same velocity components are used for all species. Toward HD206267C, for example, CN, CH, $\mathrm{CH}^{+}, \mathrm{Ca}$ I, and $\mathrm{Ca}$ II all have components at about $-3.1 \mathrm{~km} \mathrm{~s}^{-1}$ and $0.9 \mathrm{~km} \mathrm{~s}^{-1}$. The adopted velocity components for each species are indicated in Fig. 1 and 2.

Each value of $W_{\lambda}$ for the $\mathrm{CH}$ and $\mathrm{CN}$ components was converted into a column density using curves of growth. The Doppler parameter (b-value) was set at $1.0 \mathrm{~km} \mathrm{~s}^{-1}$ (Federman et al. 1994), a value consistent with the measured line widths. The inferred optical depths at line center were found to be modest $(\leq 1)$. Therefore, our column densities are not susceptible to unresolved structure. Moreover, even for a $b$-value of $0.5 \mathrm{~km} \mathrm{~s}^{-1}$, the column density ratio between sight lines for a given velocity component - from which the density contrasts are inferred - changes by only $15 \%$. When only the R(0) line of CN at $3874.61 \AA$ was detected for a component, the column density $N(\mathrm{CN})$ for the component was set equal to 1.5 times the value from $\mathrm{R}(0)$, as would be expected for an excitation temperature of 
about $2.7 \mathrm{~K}$. If a $\mathrm{CH}$ component was detected but with no corresponding $\mathrm{CN}$ absorption, a 3- $\sigma$ detection limit is given. Table 2 lists the column densities for the HD206267 system, where we find significant variations in these quantities; a complete listing of results will appear elsewhere.

\section{Analysis and Results}

\subsection{Spectral Appearance}

Two members of multiple star system HD206267 (C/D) were observed with the same instrumental setup. As shown in Figure 1, there are striking differences in the $\mathrm{CN}, \mathrm{CH}$, $\mathrm{CH}^{+}$, and $\mathrm{Ca} \mathrm{I}$ absorption profiles between the two sight lines. In addition to differences in component strengths, the Ca I profiles appear slightly "shifted" with respect to one another. Because $\mathrm{CH}^{+}$and $\mathrm{Ca}$ I absorption lines occur in the same echelle order, because the wavelength solutions for the two stars are the same, and because the $\mathrm{CH}^{+}$profiles toward the two stars match so well in velocity space, we conclude that the "shift" cannot be attributed to wavelength calibration. Since a slightly different component structure was required to get good fits for the $\mathrm{CN}$ and $\mathrm{CH}^{+}$profiles for the two sight lines, the "shift" is more likely the result of slightly different component structure.

For comparison, the slightly higher resolution $\mathrm{KPNO}$ spectra of $\mathrm{CN}, \mathrm{CH}$, and Ca II toward HD206267A were smoothed to the effective resolution of the spectra for HD206267C/D. While Gaussian fits show that the interstellar absorption toward A and C exhibits the same component structure, large variations in line strength are found for both $\mathrm{CN}$ and CH. Significant variations are also seen between the spectra toward C and D for all observed species except Ca II. It is noteworthy that $\mathrm{CN}$, which is a good tracer of dense gas (e.g., Federman et al. 1994), shows the largest variations.

Figure 2 shows significant differences in the $\mathrm{CH}^{+}$absorption profiles for the two members of the HD217035 system, but the CH, Ca I, and Ca II profiles are very similar. Absorption from $\mathrm{CN}$ was not detected in either sight line. $\mathrm{The}^{\mathrm{CH}}{ }^{+}$column densities (based on a curve of growth with $b$ of $2.5 \mathrm{~km} \mathrm{~s}^{-1}$ ) toward $\mathrm{B}$ are greater than the corresponding ones toward A by factors of 2.5, 1.5, 1.3, and 1.1 for the components at $-14.3,5.5,-10.5$, and $-6.7 \mathrm{~km} \mathrm{~s}^{-1}$, respectively. In contrast, the $\mathrm{CH}$ and $\mathrm{Ca}$ I column densities for all components show no variations between the two sight lines at the $10 \%$ level. 


\subsection{Chemical Modeling}

The significance of the line profile variations in Figures 1 and 2 and the column density variations seen in Table 2 is revealed through a simplified chemical model (Federman et al. 1994; Knauth et al. 2001), which provides estimates for the gas density in each component. The chemistry of the simple molecular species, $\mathrm{CH}, \mathrm{C}_{2}$, and $\mathrm{CN}$, is relatively well understood (van Dishoeck \& Black 1986; Federman et al. 1994); we use the formalism of Federman et al. (1994) in our analysis.

The fractional abundance of $\mathrm{C}^{+}$in diffuse gas, $x\left(\mathrm{C}^{+}\right) \sim 1.4 \times 10^{-4}$ (Meyer et al. 1997), is smaller by a factor of 1.8 than the value adopted by us in earlier studies (Federman \& Huntress 1989; Federman et al. 1994). In order to preserve the rates needed to reproduce observations of $\mathrm{C}_{2}$ and $\mathrm{CN}$, the rate coefficients, $k_{1}$ (for $\mathrm{C}^{+}+\mathrm{CH} \rightarrow \mathrm{C}_{2}^{+}+\mathrm{H}$ ) and $k_{5}$ (for $\mathrm{C}^{+}+\mathrm{NH} \rightarrow$ all products), were correspondingly increased to $5.4 \times 10^{-10}$ and $5.0 \times 10^{-10}$ $\mathrm{cm}^{3} \mathrm{~s}^{-1}$, respectively. In addition, the grain optical depth corresponding to the transition from $\mathrm{C}^{+}$to $\mathrm{CO}$ was increased from 2.0 to 2.3; otherwise, gas densities somewhat larger than those typically found in diffuse molecular gas are obtained. All other input parameters remain unchanged. The derived gas densities for all components are listed in Table 2. Uncertainties are based on the $\pm 1-\sigma$ ranges in observed $\mathrm{CH}$ and $\mathrm{CN}$ columns. For the components at $-3.1,-0.7$ and $0.9 \mathrm{~km} \mathrm{~s}^{-1}$, the chemical analysis provides evidence for factors of 1.5 to more than 5 changes in density as the cause of the $\mathrm{CN}$ profile variations seen in Fig. 1, but in no case do we infer densities as large as $5000 \mathrm{~cm}^{-3}$. While the $\mathrm{CN}$ excitation temperature can be used as an independent measure of the (electron) density (Black \& van Dishoeck 1991), our data are not of sufficient precision for such an analysis.

\section{Discussion}

Pervasive subparsec structure in the diffuse ISM, revealed by variations in atomic and molecular column densities, is beyond doubt. While other studies attempted to extract information on changes in physical condition related to these column density variations, the analyses were often based on the ad hoc assumption of a spherical cloud to determine the gas density structure. Moreover, the estimated densities are very sensitive to the temperature, which is generally not well known (Heiles 1997; Crawford et al. 2000). For instance, Crawford et al. (2000) used the column density ratio, $N(\mathrm{~K} \mathrm{I}) / N(\mathrm{Na} \mathrm{I})$, to estimate the physical conditions in relatively dense clouds. Toward HD81188, an increase in that ratio by a factor of 1.9 , on a scale of $\sim 15 \mathrm{AU}$, could be explained either by increasing gas density by a factor of 5 or by decreasing temperature by a factor of 10 . More likely, it may be due to some combination of density increase and temperature decrease. In many 
optical studies, an important unknown factor is the fractional ionization, which is needed to estimate $N(\mathrm{H})$ from trace neutral species such as Na I (Lauroesch et al. 1998; Welty \& Fitzpatrick 2001).

In our chemical analysis, the density is determined essentially by a column density ratio, $N(\mathrm{CN}) / N(\mathrm{CH})$, which reduces influences of cloud shape on density structure. For example, the $-3.1 \mathrm{~km} \mathrm{~s}^{-1}$ component toward HD206267C and the $-0.7 \mathrm{~km} \mathrm{~s}^{-1}$ one toward HD206267D have similar $N(\mathrm{CN}) / N(\mathrm{CH})$ - and thus comparable gas densities - even though their $\mathrm{CN}$ and $\mathrm{CH}$ column densities differ by a factor of about 3. Our derived gas density also is not sensitive to temperature. For instance, calculated densities for velocity components toward $\mathrm{A}$ and $\mathrm{C}$ increase by only 3 to $8 \%$ when the temperature of $40 \mathrm{~K}$ is halved. The change in gas density ratios for a given velocity component in the two sight lines is even smaller, $1-2 \%$, when the temperature is halved.

Calculations also show that the gas density ratio for a given velocity component is not overly sensitive to the reaction rates in the chemical model. The rate coefficients $k_{1}$ and $k_{5}$ produce the largest effects on the gas density; decreasing either increases the calculated density. When both rate coefficients are decreased by $80 \%$, the derived gas densities toward HD206267A and HD206267C increase by 50-80\%, but the gas density ratios for individual velocity components in the two sightlines change by only $4-8 \%$. The greatest change in this density ratio (from 1.58 to 1.72 ) is found for the $-0.7 \mathrm{~km} \mathrm{~s}^{-1}$ component. We conclude that this chemical analysis is a good approach for deriving physical properties of small scale structure in diffuse gas clouds with detectable amounts of $\mathrm{CN}$.

The HD206267 multiple star system is located in a region of recent, active star formation. LM99 studied K I variations toward members of this system, and estimated typical gas density ratios of 1.1-2.7 for velocity components toward A and C. [Here we adopt $N(\mathrm{~K} \mathrm{I}) \propto n^{2.7}$ instead of $n^{1.7}$ to take into account the additional factor of density in $N(\mathrm{~K}$ II).] The density ratios derived from $\mathrm{CH}$ and $\mathrm{CN}$ range from 1.5 to greater than 5.0. The lower density ratios found by LM99 presumably can be attributed to K I absorption probing a more extended volume of the cloud than CN absorption. The larger volume likely smooths out the large density variations in CN-rich gas. This picture is consistent with our Ca II observations, which show no significant profile variations, probably because Ca II absorption occurs over an even more extended region (Crinklaw, Federman, \& Joseph 1994; Welty, Morton, \& Hobbs 1996). The present investigation also supports the conjecture of LM99 that much of the variation toward this system may be in dense gas. Table 2 shows that the gas density variations among sight lines of the system are detected only in components with $v_{L S R}$ from -4.0 to $1.0 \mathrm{~km} \mathrm{~s}^{-1}$, with no obvious large density variation for the $4.3 \mathrm{~km} \mathrm{~s}^{-1}$ component. The CO emission-line maps of Patel et al. (1998) indicate that 
CO-rich material in these lines of sight only appears within a velocity range of $v_{L S R} \sim-5.0$ $\mathrm{km} \mathrm{s}^{-1}$ to $\sim 2.0 \mathrm{~km} \mathrm{~s}^{-1}$ - the range in which we see significant variations. Furthermore, using the K I results of LM99 with the trends determined by Welty and Hobbs (2001) for $\mathrm{H}_{t o t}$, we find $\mathrm{H}_{t o t}$ of $\approx 1 \times 10^{21} \mathrm{~cm}^{-2}$ for these components and only about $4 \times 10^{20} \mathrm{~cm}^{-2}$ for the $+4.3 \mathrm{~km} \mathrm{~s}^{-1}$ one. Our gas densities then suggest cloud thicknesses of about $1 \mathrm{pc}$ along the lines of sight, similar to the sizes of the CO cloudlets seen in emission (Patel et al. 1998).

Variations in $\mathrm{CN}$ and $\mathrm{CH}$ column densities were not detected toward the HD217035 system. Since only upper limits for CN are available for these sight lines, the density must be relatively low. The variations in the $\mathrm{CH}^{+}$profiles toward both systems may help to elucidate the uncertain chemistry for this molecule.

In summary, our observations of $\mathrm{CN}$ and $\mathrm{CH}$ absorption show that the diffuse molecular clouds toward the multiple star system HD206267 exhibit significant density structure. The gas density varies by factors greater than 5.0 over a scale of $\sim 10,000 \mathrm{AU}$. The present approach to studying small-scale structure in diffuse clouds provides reliable density contrasts, but it is limited to relatively dense molecule-rich clouds.

It is our pleasure to thank the support staff of McDonald Observatory and KPNO, especially David Doss at McDonald Observatory. This work was supported by NASA grants NAG5-4957 and NAG5-8961 to the University of Toledo.

\section{REFERENCES}

Andrews, S.M., Meyer, D.M., \& Lauroesch, J.T. 2001, ApJ, 552, L73

Black, J.H., \& van Dishoeck, E.F. 1991, ApJ, 369, L9

Bates, B., Shaw, C.R., Kemp, S.N., Keenan, F.P., \& Davies, R.D. 1995, ApJ, 444, 672

Crawford, D.L., \& Barnes, J.V. 1970, AJ, 75, 952

Crawford, I.A., Howarth, I.D., Ryder, S.D., \& Stathakis, R.A. 2000, MNRAS, 319, L1

Crinklaw, G., Federman, S.R., \&Joseph, C.L. 1994, ApJ, 424, 748

de Zeeuw, P.T., Hoogerwerf, R., de Bruijne, J.H.J., Brown, A.G.A. \& Blaauw, A. 1999, AJ, 117,354

Dommanget, J., \& Nys, O. 1994, Bull. Infor. Centre Donnees Stellaires, 45, 7

ESA 1997, The Hipparcos and Tycho Catalogues, ESA SP-1200, ESA Publications Division Noordwijk 
Faison, M.D., \& Goss, W.M. 2001, AJ, 121, 2706

Federman, S.R., \& Huntress, W.T. 1989, ApJ, 338, 140

Federman, S.R., Strom, C.J., Lambert, D.L., Cardelli, J.A., Smith, V.V., \& Joseph, C.L. 1994, ApJ, 424, 772

Frail, D.A., Weisberg, J.M., Cordes, J.M., \& Mathers, C. 1994, ApJ, 436, 144

Heiles, C. 1997, ApJ, 481193

Knauth, D.C., Federman, S.R., Pan, K., Yan, M., \& Lambert, D.L. 2001, ApJS, 135, 201

Langer, G.E., Prosser, C.F., \& Sneden, C. 1990, AJ, 100, 216

Lauroesch, J.T., \& Meyer, D.M. 1999, ApJ, 519, L181-LM99

Lauroesch, J.T., Meyer, D.M., \& Blades, J.C. 2000, ApJ, 543, L43

Lauroesch, J.T., Meyer, D.M., Watson, J.K, \& Blades, J.C. 1998, ApJ, 507, L89

Meyer, D.M., \& Blades, J.C. 1996, ApJ, 464, L179

Meyer, D.M., Cardelli, J.,A., \& Sofia, U.J. 1997, ApJ, 490, 103

Meyer, D.M., \& Lauroesch, J.T. 1999, ApJ, 520, L103

Moore, E.M., \& Marscher, A.P. 1995, ApJ, 452, 671

Patel N.A., Goldsmith P.F., Heyer M.H., Snell, R.L., \& Pratap, R. 1998, ApJ, 507, 241

Price, R.J., Crawford, I.A., \& Barlow, M.J. 2000, MNRAS, 312, L43

Tull, R.G., MacQueen, P.J., Sneden, C., \& Lambert, D.L. 1995, PASP, 107, 251

van Dishoeck, E.F., \& Black, J. H. 1986, ApJS, 62109

Watson, J.K., \&Meyer, D.M. 1996, ApJ, 473, L127

Welty, D.E., \& Fitzpatrick, E.L. 2001, ApJ, 551, L75

Welty, D.E., \& Hobbs, L.M. 2001, ApJS, 133, 345

Welty, D.E., Morton, D.C., \& Hobbs, L.M. 1996, ApJS, 106, 533 
Table 1. Stellar Data

\begin{tabular}{|c|c|c|c|c|c|c|}
\hline \multirow[t]{2}{*}{ System } & \multirow{2}{*}{$\begin{array}{c}V \\
(\mathrm{mag})\end{array}$} & \multirow{2}{*}{$\begin{array}{c}E(B-V) \\
\quad(\mathrm{mag})\end{array}$} & \multirow{2}{*}{$\begin{array}{c}D \\
(\mathrm{pc})\end{array}$} & \multicolumn{2}{|c|}{ Separation } & \multirow[t]{2}{*}{ Reference } \\
\hline & & & & $(\operatorname{arcsec})$ & $(\mathrm{AU})$ & \\
\hline \multirow[t]{3}{*}{ HD206267A/C/D } & $5.7 / 8.0 / 7.9$ & $0.52 / 0.57 / 0.42$ & $615 \pm 15$ & AC: 11.8 & 7,250 & 1,2 \\
\hline & & & & CD: 31.6 & 19,410 & \\
\hline & & & & $\mathrm{AD}: 19.9$ & 12,220 & \\
\hline HD217035A/B & $8.6 / 8.6$ & $0.76 / 0.76$ & 725 & 1.9 & 1,380 & 3,4 \\
\hline
\end{tabular}

References. - (1) ESA 1997; (2) de Zeeuw et al. 1999; (3) Crawford \& Barnes 1970; (4) Dommanget \& Nys 1994.

Table 2. Column Densities and Densities for the HD206267 System

\begin{tabular}{lcccccc}
\hline \hline \multirow{2}{*}{ Sight Line } & \multirow{2}{*}{ Quantity } & \multicolumn{5}{c}{$v_{L S R}\left(\mathrm{~km} \mathrm{~s}^{-1}\right)$} \\
\cline { 3 - 7 } & & -4.1 & -3.1 & -0.7 & 0.9 & 4.3 \\
\hline \multirow{2}{*}{ HD206267A } & $N(\mathrm{CN})\left(10^{12} \mathrm{~cm}^{-2}\right)$ & $\ldots$ & $7.0 \pm 0.2$ & $0.9 \pm 0.2$ & $<0.2$ & $<0.2$ \\
& $N(\mathrm{CH})\left(10^{12} \mathrm{~cm}^{-2}\right)$ & $\ldots$ & $14.8 \pm 0.5$ & $3.6 \pm 0.3$ & $10.8 \pm 0.5$ & $3.5 \pm 0.3$ \\
& $n\left(\mathrm{~cm}^{-3}\right)$ & $\ldots$ & $1500 \pm 110$ & $700 \pm 120$ & $<60$ & $<170$ \\
HD206267C & $N(\mathrm{CN})\left(10^{12} \mathrm{~cm}^{-2}\right)$ & $\ldots$ & $4.9 \pm 0.3$ & $0.9 \pm 0.2$ & $1.8 \pm 0.3$ & $<0.3$ \\
& $N(\mathrm{CH})\left(10^{12} \mathrm{~cm}^{-2}\right)$ & $\ldots$ & $12.5 \pm 0.8$ & $2.4 \pm 0.5$ & $10.7 \pm 0.8$ & $3.8 \pm 0.6$ \\
& $n\left(\mathrm{~cm}^{-3}\right)$ & $\ldots$ & $1000 \pm 200$ & $1100 \pm 500$ & $400 \pm 90$ & $<180$ \\
HD206267D & $N(\mathrm{CN})\left(10^{12} \mathrm{~cm}^{-2}\right)$ & $1.3 \pm 0.2$ & $\ldots$ & $1.4 \pm 0.2$ & $<0.3$ & $<0.3$ \\
& $N(\mathrm{CH})\left(10^{12} \mathrm{~cm}^{-2}\right)$ & $6.7 \pm 0.4$ & $\ldots$ & $4.5 \pm 0.4$ & $8.0 \pm 0.4$ & $7.1 \pm 0.5$ \\
& $n\left(\mathrm{~cm}^{-3}\right)$ & $600 \pm 130$ & $\ldots$ & $950 \pm 220$ & $<130$ & $<150$ \\
\hline \hline
\end{tabular}




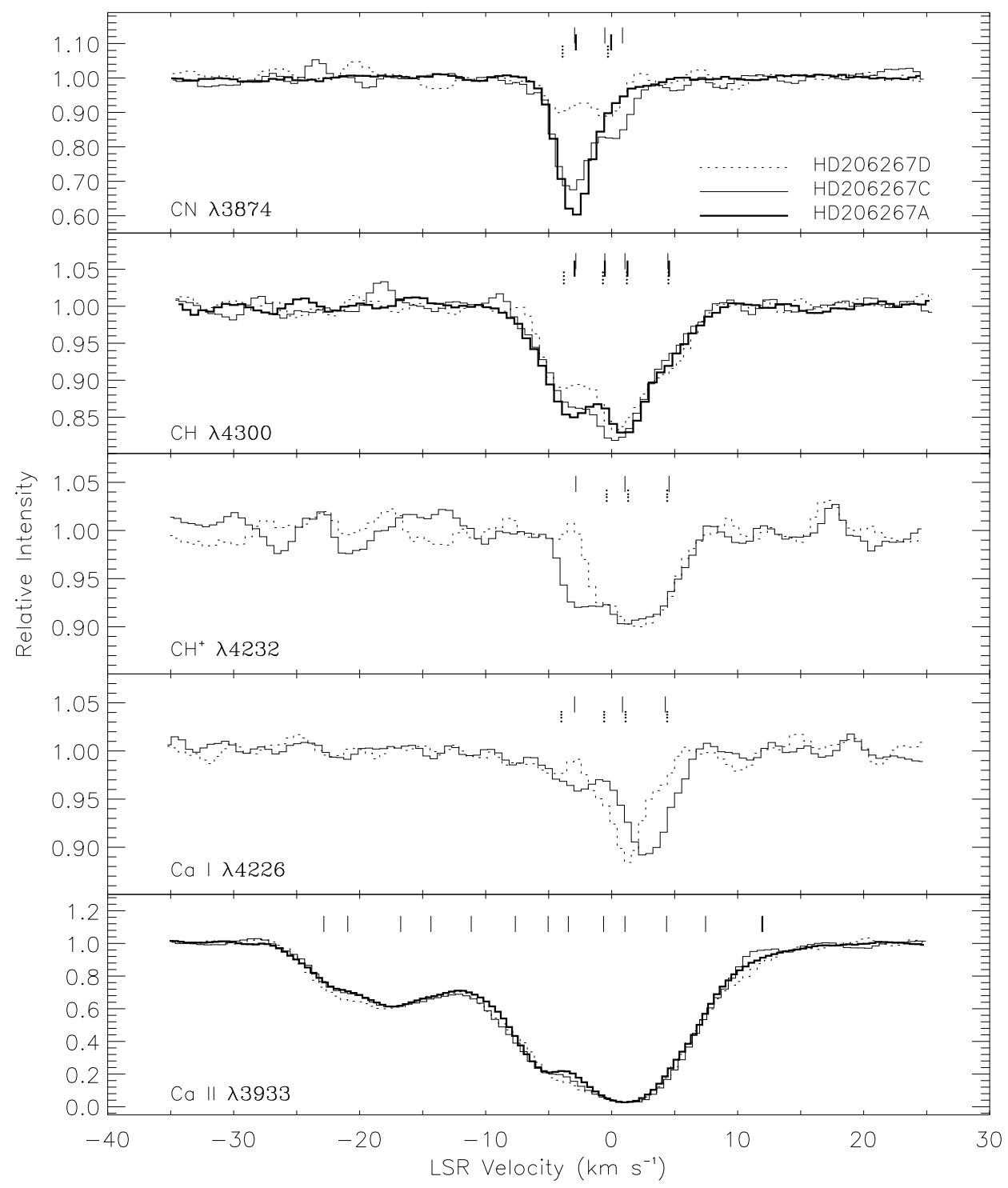

Fig. 1.- Absorption from interstellar $\mathrm{CN}, \mathrm{CH}, \mathrm{CH}^{+}, \mathrm{Ca} \mathrm{I}$, and $\mathrm{Ca}$ II in the spectra of HD206267A (thick solid lines), HD206267C (solid lines) and HD206267D (dotted lines). The observed line is indicated. Note the relative intensity scales for the panels differ. Hash marks above each spectrum show the component structure derived from Gaussian fits. 


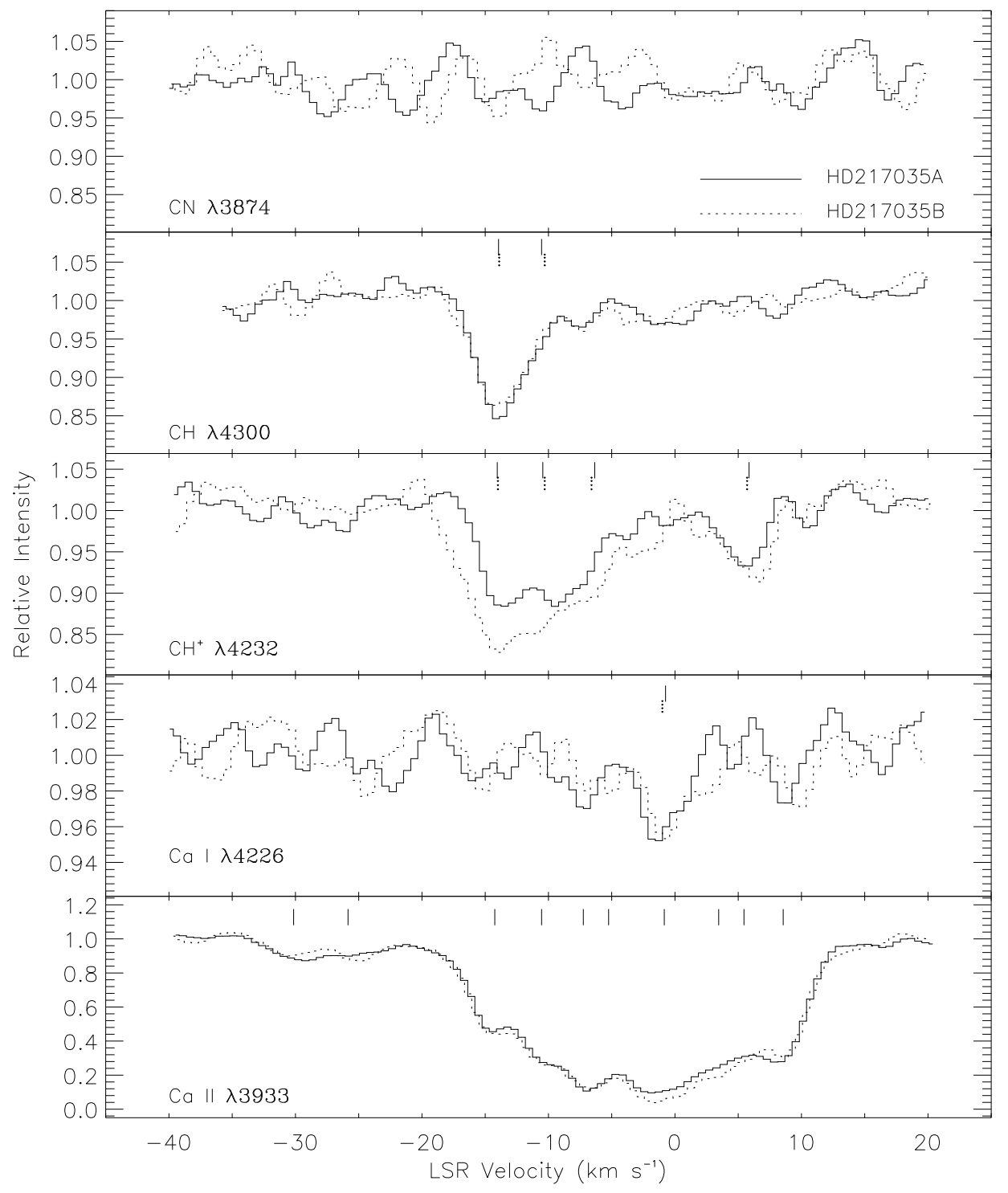

Fig. 2.- Same as Fig. 1 for HD217035A (solid lines) and HD217035B (dotted lines). 